\title{
New Approach of Hand writing Recognition using Curvelet Transform and Invariant Statistical Features
}

\author{
Pankaj Kumawat \\ M.E.computer science and \\ engineering, Medicaps Institute of \\ Technology, Indore (India)
}

\author{
Asha Khatri \\ Department of computer science \\ and engineering, Medicaps Institute \\ of Technology, Indore (India)
}

\author{
Baluram Nagaria \\ Department of Electronics \& \\ Communication Engineering \\ MIT, Mandsaur (India)
}

\begin{abstract}
The work, as mentioned can be used in the forensic studies for identifying the authenticity of a person. We can use the work in age analysis of the person from his writing. Further the efficiency of the system is very high in comparison to the existing system. The work is to recognize handwriting of a person by using hidden markov model, support vector machine and our new approach HMM-SVM classifier using MATLAB. In previous HMM and SVM classifier are used to the handwriting recognition. In this paper we used the combined features of HMM and SVM classifier using combination of Curvelet transform and Invariant transform. Performance of the system depends entirely on the feature vectors. Further we compare the performance of HMM, based technique with combined HMM- SVM based technique and found that for some combined HMM-SVM technique is better than HMM. Only curvelet transform using HMM or SVM get good accuracy but for better accuracy required the combined HMM-SVM classifier. It improve the problem of HMM classifier of multiple detection of Class to. However a kernel based technique adds advantage over probabilistic methods in certain deterministic states. Hence we combine both HMM and SVM to design a combined classifier for the problem. We have also evaluate the performance of HMM, SVM and Combined HMM-SVM classifier.
\end{abstract}

\section{Keywords}

Hidden markov model (HMM), state vector machine-SVM, HMM-SVM classifier, Curvelet transform (CT) Invariant Statistical Features (IFS), Thresholding

\section{INTRODUCTION}

A person's handwriting - the script - and its placing on the page express the unique impulses of the individual: logically, the brain sends signals along the muscles to the writing implement they control. By examining a handwriting sample, an expert graphologist is able to identify relevant features of the handwritten script, and the way the features interact. The features, and interaction between them, provide the information for the analysis. We define some of the common analysis models and then propose their corresponding image processing modeling and machine learning in the methodology section. [7] No single handwriting feature proves anything specific or absolute by itself; a single feature alone can only identify a trend. It is the combination of features, and the interaction between them that enable a full and clear interpretation.perform binarization and extract invariant moments from the image document followed by the curvelet transform. These features are used to build a HMM model which we use to classify the features of a test document.[3] It can be extended in writing biometric where a person can be authenticated based on his age. Biometrics is the science of verifying the identity of an individual through physiological measurements or behavioral traits. Since biometric identifiers are associated permanently with the user they are more reliable than token or knowledge based authentication methods. Biometrics offers several advantages over traditional security measures.[5] These includes

1. Non-repudiation

2. Accuracy and Security

3. Screening

A number of biometric identifiers are in use in various applications. Each biometric has its strengths and weaknesses and the choice typically depends on the application.

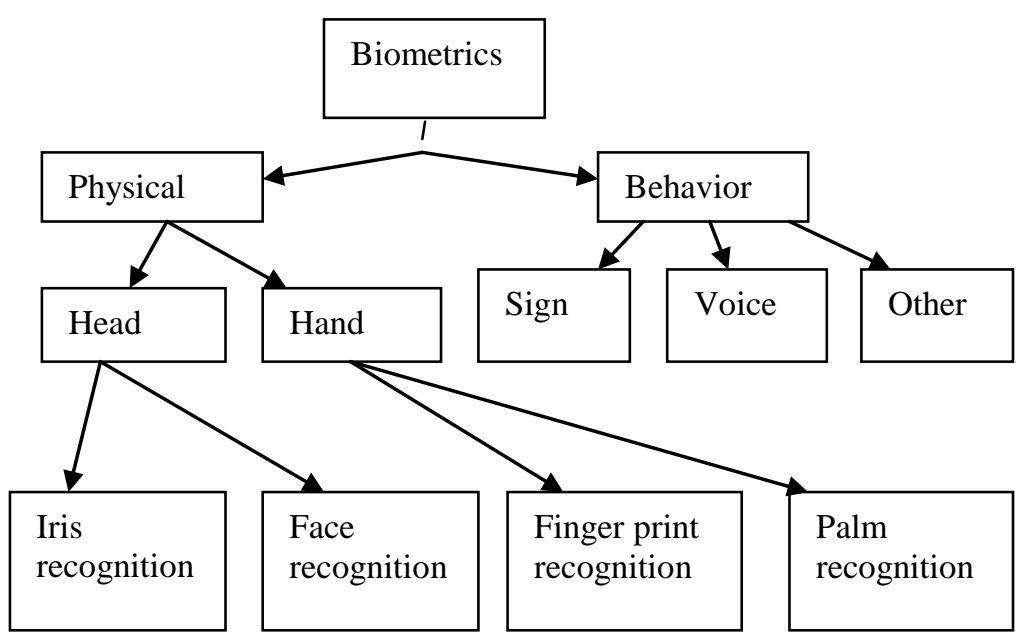

Figure 1: various biometric of hand writing recognition

\section{SCOPE OF THE PROJECT}

The work, as mentioned can be used in the identifying the authenticity of a person. The technique can also be used in handwritten character recognition. Handwriting recognition using different techniques is used to analysis handwritten signature verification and optical character recognition. Further the efficiency of the system is very high in comparison to the existing system.

\section{PROBLEM WITH THE PRESENT SYSTEM AND RECENT SCENARIO}

The statement of the work can be simply put as to recognize the handwriting of the person by using HMM. First person' 
handwriting are written on white paper which are scanned and given as input to the system. The input comprises of set of characters, say a line of text. System extracts the features and creates a feature database. When a sample scanned image of a person is given as input, it identifies the writer. As can be seen from the above description that all the above mentioned feature are time dependent, writing dependent, Scanner Quality dependent. Hence these can not extract the person dependent features which are consistent irrespective of the mentioned constraints. Hence we propose HMM based features which are independent of these constraints.

In 2006, Roman Bertolami et al [14]' Rejection strategies for offline handwritten text line recognition. This paper investigates rejection strategies for unconstrained offline handwritten text line recognition. The rejection strategies depend on various confidence measures that are based on alternative word sequences. The alternative word sequences are derived from specific integration of a statistical language model in the hidden Markov model based recognition system.

In 2009, Yousri KESSENTINI et al.[12]' Off-Line Handwritten Word Recognition Using Multi-Stream Hidden Markov Models are discussed. This paper, present a multi-stream approach for off-line handwritten word recognition. The multi-stream paradigm provides an interesting framework for the integration of multiple sources of information and is compared to the standard combination strategies namely fusion of representations and fusion of decisions. This investigate the extension of 2stream approach to $N$ streams $(N=2, \ldots, 4)$ and analyze the improvement in the recognition performance. Significant experiments have been carried out on two publicly available word databases: IFN/ENIT benchmark database (Arabic script) and IRONOFF database (Latin script). The multi-stream framework improves the recognition performance in both cases. Using 2-stream approach, the best recognition performance is $79.8 \%$, in the case of the Arabicscript, on a 2100-word lexicon consisting of 946 Tunisian town/village names. In the case ofthe Latin script, the proposed approach achieves a recognition rate of $89.8 \%$ using a lexiconof 196 words. The statement of the work can be simply put as to recognize the handwriting of the person by using HMM. First person' handwriting are written on white paper which are scanned and given as input to the system. The input comprises of set of characters, say a line of text. System extracts the features and creates a feature database. When a sample scanned image of a person is given as input, it identifies the writer.

'In 2010, Rahul KALA et al [13]' Handwriting reorganization by using genetic algorithmIn this algorithm we will take a deep insight into the algorithm and its working. We discuss about the handwriting recognition general procedure, the algorithmic assumptions and its working. We know that we are given an unknown character that needs to be recognized. For this we have diverse form of training data available for each and every

character. In this algorithm we try to match the input to the training data and the data generated from intermixing of training data, to find the best match for the given input data. In this paper problem identified that It required lot of tanning data sample to get good efficiency and also recognize straight line characters' only this algorithm cannot recognize the character are tilled some left or right side.

\section{PERFORMANCE MEASUREMENT OF HANDWRITING RECOGNITION}

There are some basic measurements for Handwriting recognition techniques some handwriting features there are introduce in article to explain some of the basic ones that can be readily understood and which give their information.[10] features such as slant, size, pressure, upper zone, middle zone, lower zone, word spacing, line spacing etc given bellow.[11]

Slant: Right slant indicates a response to communication, but not how it takes place.

Size: A basic average measure - or benchmark - by which size can be judged is $3 \mathrm{~mm}$ per zone. This gives a benchmark for a non-remarkable full height of $9 \mathrm{~mm}$.

Pressure: Heavy pressure indicates commitment and taking things seriously, but if the pressure is excessively heavy, that writer gets very uptight at times and can react quickly to what they might see as criticism, even though none may have been intended.

Upper zone: In this case In $1, \mathrm{t}, \mathrm{h}$, etc are used Tall upper strokes are reaching towards goals and ambitions or, if they are very extended, there may be unrealistic expectations of what the person feels they must achieve.

Middle zone: In this case a, c, e, etc are used. These middle zone shapes can give some particularly interesting information.

Lower zone: In this case g, y, p, etc are used. Lower loops are also varied and have different meanings.

Word spacing: The benchmark by which to judge wide or narrow spacing between words is the width of one letter of the person's handwriting.

Line spacing: Handwriting samples are always best on unlined paper, and particularly for exhibiting line-spacing features. Wide-spaced lines of handwriting show a wish to stand back and take a long view. Closely spaced lines indicate that that the writer operates close to the action.

Page margins: The sides of the page each have a meaning. The left side margin shows the roots and beginnings/family. The right side shows other people and the future. The top is goals and ambitions. The foot of the page shows energy, instincts and practicality. [11]

\section{DIFFERNENT TECHNIQUES Statistical Classification}

Statistical classification is the problem of identifying which of a set of categories a new observation belongs, on the basis of a training set of data containing observations whose category membership is known. In case of statistical approach, each pattern is viewed as a point in the d-dimensional space. Features are chosen so that disjoint regions in a dimensional feature space are occupied by the pattern vectors belonging to different categories [17]. Decision boundaries are established in the feature space to separate the patterns belonging to different classes. Parametric and non-parametric are the two classifications of statistical methods. Parametric methods are the one in which handwriting samples are categorized using a set of parameters which are selected based on the training data. Nonparametric methods are based on direct estimation from the training data.

HMM i.e. Hidden Markov Models, k-NN i.e. k-Nearest Neighbors and SVM i.e. Support Vector Machines are few important examples of statistical recognition.[17]

\section{(a).Hidden Markov Model}

Hidden Markov Models (HMMs) are powerful statistical models for modeling sequential or time-series data, and have been successfully used in many tasks such as speech recognition, protein/DNA sequence analysis, robot control, and information 
extraction from text data.[15] There are two different formulations of HMMs depending on whether to Igenerate" the output from a state or a transition. As a probabilistic model, there is no essential difference, though. We assume that an output symbol is always generated from a state and that any state can be a starting state or ending state. First we determine the probability matrix of appearance of 1 with respect to 0 and 1 and probability of occurrence of 0 with respect to 1 and 0 . This pattern is unique for all the signatures. As the signatures are considered random in nature, we consider a hidden markov model that can generate such a pattern. The resulting series of the velocity values for each probability serves as the sequence of observationsymbols for training a discrete HMM. As outlined in Section 1, a HMM based signature verification system typically involves two phases - training and verification. [3]

The three problems associated with an HMM are:[15]

Evaluation: Evaluating the probability of an observed sequence of symbols

Decoding: Finding the most likely state transition path associated with an observed sequence.

Training: Adjusting all the parameters to maximize the probability of generating an observed sequence.

Three basic algorithm are used to solve above problems [16]

Forward and backward Algorithm: Evaluation is solved by using the forward and backward iterative algorithms.

Viterbi algorithm: Decoding is solved by using the Viterbi algorithm, also an iterative algorithm to grow the best path by sequentially considering each observed symbol.

Baum-Welch algorithm (an EM algorithm): The last Training is solved by the Baum-Welch algorithm (an EM algorithm), which uses the forward and backward probabilities to update the parameters iteratively. Below, we give the formulas for each of the step of the computation.

\section{(b). Support Vector Machines (SVM):}

SVM are binary classifiers that separate linearly any two classes by finding a hyperplane of maximum margin between the two classes. The margin means the minimal distance from the separating hyperplane to the closest data points. SVM learning machines searches for an optimal separating hyperplane where the margin is maximal. The outcome of the SVM is based only on the data points that are at the margin and are called,,Support Vectors" [17]. Used mostly for classification (also, can be modified for regression and even for unsupervised learning applications). Achieve accuracy comparable to Multilayer Perceptions [4]

\section{Classification of SVM Kernels[18]}

Linear kernel: Linear SVM is linearly scalable with the size of the training data set. It is given by the following formula:

$$
\mathrm{K}(\mathrm{x}, \mathrm{y})=\mathrm{x} * \mathrm{y}
$$

Where, $\mathrm{k}(\mathrm{x}, \mathrm{y})$ is the kernel function

Polynomial kernel: It is a non-stationary kernel which is well suited for problems where the training data is normalized. It is given by the following formula:

$$
\mathrm{K}(\mathrm{x}, \mathrm{y})=\left(\mathrm{a} \mathrm{x}^{\mathrm{y}} \mathrm{y}=\mathrm{c}\right)^{\mathrm{d}}
$$

Where, $\alpha$ is the slope, $c$ is the constant term and $d$ is the polynomial degree

RBF kernel: It is defined on the interval $[-1,1]$ and is given by the following formula:

$$
\mathrm{K}(\mathrm{x}, \mathrm{y})=\exp \left(\square\|\mathrm{x} \square \mathrm{y}\|^{2} / 2 \sigma^{2}\right)
$$

Sigmoid kernel: With gain $\kappa$ and offset $\Theta$, the formula for a sigmoid kernel is given by:

$$
K(x, y)=\tan \mathrm{h}(\kappa(x, y)+\theta)
$$

(c). HMM-SVM Classifier: This is the combination of HMM and SVM techniques using the combined features of curvelet transform and Invariant Statistical Features (IFS) and this is our new approach.

\section{PROPOSED MYTHOLOGY}

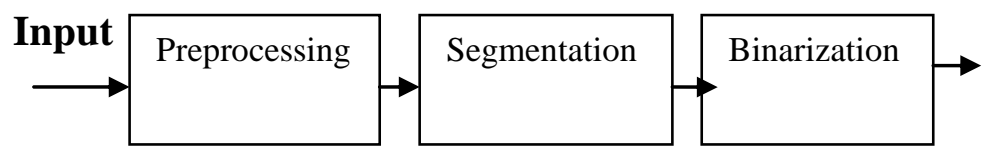

Skew correction/norm

Natural

Thresholding.

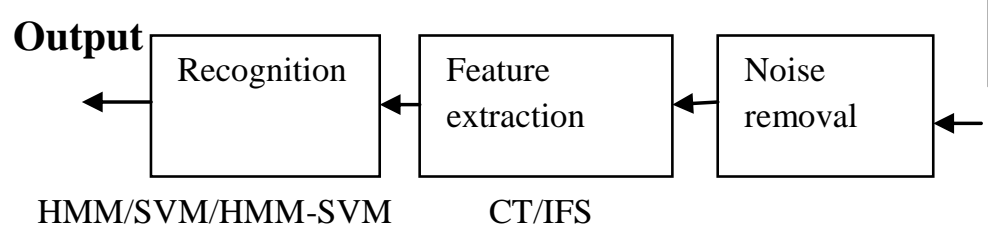

Figure: 2 proposed block diagram of hand writing
recognition

Preprocessing: The first step to any document analysis is preprocessing. The steps are elaborated bellow. First the sample text is scanned and stored as an image. This is specifically a gray scale image. A binary image is generated out of this gray scale image and one bit binary noise is removed by using erosion.[5] Segmentation: A simple ligature model is used; the features used to detect ligatures are very simple to detect too.

Binarization:. All gray scale images are binarized with the help of Natural Thresholding. In this process the mean gray level value of the images are extracted and values greater than the mean are mapped to white where as the values lesser than that are marked as black.[5]

Noise Removal:Once we have the binarized image a simple $5 \mathrm{X} 5$ morphological filter is applied to clean the spatial noise characterized by small components of size ( 1 to 3 pixels). This clean binarized image is cropped from both the directions. The image is further Feature Extraction[4]

Feature extraction: Read the matrices from image after preprocessing, Extract HMM features and Extract statistical data from HMM states like mean and standard deviationadded on all the four sides[7]

Recognition: Each state now represents a matrix which can define a probability matrix as calculated prior to HMM.The statistical mean and standard deviation of each value is taken as the training vectors for a SVM.[7]

\section{SIMULATION RESULT OF VARIOS TECHNIQUES}

This study presented an analysis and comparison of handwriting recognition usingHMM, SVM\&combined HMM-SVM classifier. The accuracy of combined HMM-SVM classifier is better than other two techniques with the combined features of curvelet transform and Invariant Statistical Features (IFS).we have taken 200 samples of two user and applying three handwriting techniques and concluded that HMM have low accuracy with curvelet transform ,SVM has better than HMM but it apply a large amount of data .our newtechniques Combined HMM-SVM with using both curvelet transform and Invariant Statistical Features gives more accuracy than the other two techniques with very less number of sample. as well as high number of samples.

Hidden markov model is a probabilistic representation of states. Hence HMM is most preferred technique in this domain. It is 
due to the fact the HMM produces good result for large number of statistical patterns.

The values of corresponding performance parameters for handwriting recognition are tabularized as shown below

Table 7.1 Comparison of handwriting recognition with HMM, SVM and combine HMM-SVM Classifier

\begin{tabular}{|c|c|c|c|}
\hline $\begin{array}{l}\text { number } \\
\text { of } \\
\text { sample }\end{array}$ & SVM & HMM & $\mathrm{HMM}+\mathrm{SVM}$ \\
\hline 10 & \multirow{20}{*}{$\begin{array}{l}\text { Accuracy of } \\
\text { svm of } \\
\text { whole } 200 \\
\text { samples }\end{array}$} & 90 & 90 \\
\hline 20 & & 94.73 & 94.85 \\
\hline 30 & & 96.29 & 96.75 \\
\hline 40 & & 97.22 & 97.29 \\
\hline 50 & & 97.86 & 97.87 \\
\hline 60 & & 96.42 & 98.24 \\
\hline 70 & & 95.45 & 98.5 \\
\hline 80 & & 97.33 & 98.6 \\
\hline 90 & & 97.66 & 98.83 \\
\hline 100 & & 96.8 & 98.94 \\
\hline 110 & & 97.08 & 99 \\
\hline 120 & & 97.32 & 99.11 \\
\hline 130 & & 96.69 & 98.36 \\
\hline 140 & & 96.18 & 98.48 \\
\hline 150 & & 96.42 & 98.58 \\
\hline 160 & & 96.66 & 98.66 \\
\hline 170 & & 96.83 & 98.73 \\
\hline 180 & & 96.98 & 98.87 \\
\hline 190 & & 97.13 & 98.87 \\
\hline 200 & & 97.31 & 98.92 \\
\hline
\end{tabular}

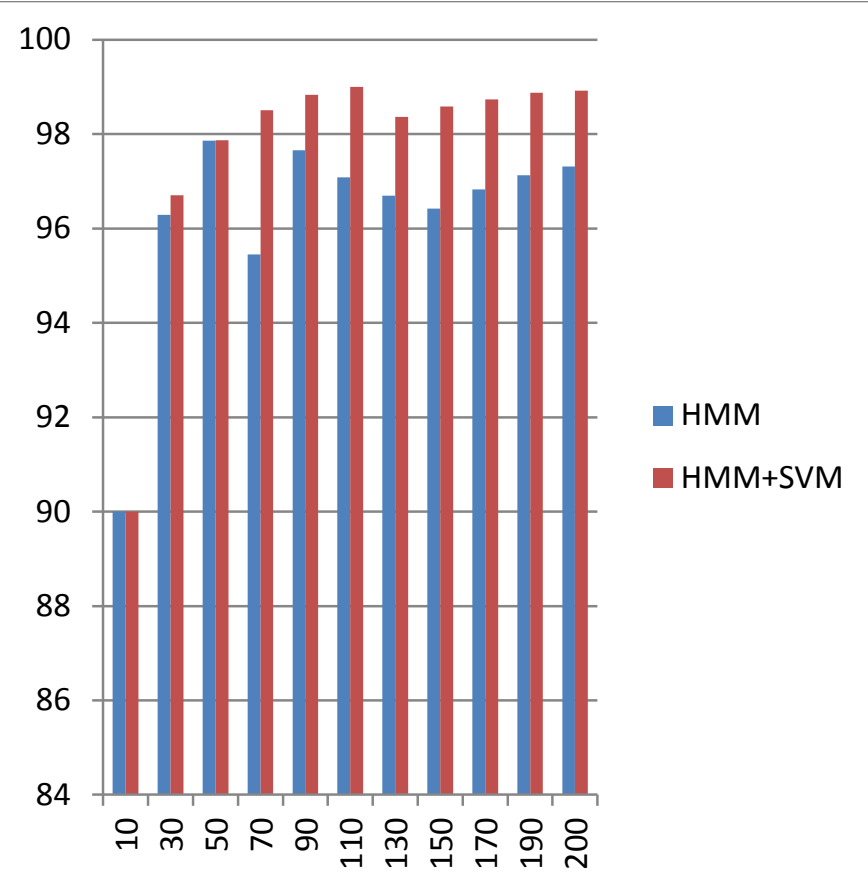

Figure: 3 comparison of HMM and combined HMM-SVM classifier between samples and accuracy

\section{CONCLUSION AND FUTURE WORK}

There are different handwriting techniques are used for recognition such s HMM, SVM and combined features of HMM-SVM classifier. There are context with respect to which a person may choose to write. It may include writing a small phrase, character, a paragraph or so. The paper on which he chooses to write may also vary. In this paper the accuracy is increase with using different types of algorithms depending on the techniques are used. Due to this the success of the algorithm largely depends upon preprocessing steps and the efficiency of preprocessing. Preprocessing technique is adopted to neutralize these constraints and is used for tilt correction, filtering of the documents etc. Hence efficiency of the system may decrease if the preprocessing result is not appropriate.Handwriting is rather a pattern associated with different users. Therefore handwriting recognition differs from other similar recognition like signature biometric. In this work we have presented a Novel technique of Handwriting recognition by first extracting statistical moments and curvelet features from the user's handwriting pattern and than forming a statistical state machine with HMM-SVM classifier. Further the technique was improved by the aid of kernel based techniques with the Help of SVM. As kernel based techniques transforms the training vector to more separable vectors, the accuracy achieved is very high. 


\section{REFERENCES}

[1] Casey R.G. and Lecolinet E., A survey of methods and strategies in character segmentation. IEEE Trans. PAMI 18 (7) 1996 pp. 690-706.

[2] Cesar M. and Shingal R., Algorithm for segmenting handwritten postal codes. Int'l J. Man Machine Studies 33 (1) 1990 pp. 63-80.

[3] Baird H.S., Kahan S. and Pavlidis T., Component of an Omnifont Page Reader. Proc 8th ICPR Paris 1986 pp. 344348.

[4] Yanikoglu B. and Sandon P.A., Segmentation of off-line cursive handwriting using linear programming. Patt.Recog. 31 (12) 1998 pp. 1825-1833.

[5] Bozinovic R.M. and Shrihari S.N., Off-line cursive script recognition.IEEE Trans. PAMI 11 (1) 1989 pp. 68-83.

[6] Kimura F. et al., Improvements of a Lexicon Directed Algorithm for Recognition of Unconstrained Handwritten Words. Proc 2nd ICDAR Tsukuba October 20-22 1993 pp. $18-22$.

[7] Romeo-Pakker K. et al. A New Approach for Latin Arabic Character Segmentation 3rd ICDAR, Montreal, August 1416,1995 , pp 874-877.

[8] David A. Katz, Handwriting Analysis

[9] Javed Ahmed Mahar,Mohammad Khalid Khan,Prof. Dr. MumtazHussainMahar," Off-Line Signature Verifi'cation of Bank Cheque Having Different Background Colors"

[10] Bikash Shaw, Swapan Kumar Parui, Malayappan Shridhar Offline Handwritten DevanagariWord Recognition: A holistic approach based on directional chain code feature and HMM
[11] Edson J. R. Justin0 FlfivioBortolozzi ', Robert Sabourin ', Off-line Signature Verification Using HMM for Random, Simple and Skilled Forgeries

[12] Yousri Kessentini, Thierry Paquet1, AbdelmajidBenhamadou' Off-Line Handwritten Word Recognition Using Multi-Stream Hidden Markov Models published in "Pattern Recognition Letters 31, 1 (2010) 60 70" DOI : 10.1016/j.patrec.2009.08.009

[13] Rahul Kala, Harsh Vazirani, AnupamShukla, RituTiwari 'Handwriting reorganization by using genetic algorithm' International Journal of Computer Science Issues, Vol. 7, Issue 2, No 1, March 2010

[14] Roman Bertolami_Matthias Zimmermann 1 Horst Bunke' Rejection strategies for offline handwritten text line recognition' ACM Portal, Vol. 27, Issue. 16, December 2006

[15] L.R. Rabiner and B.H. Juang. An introduction to hidden markov models. In IEEEASSP Magazine, 1986. pp. $4\{16$.

[16] Rabiner, L. R. (1989). A tutorial on hidden Markov models and selected applications in speech recognition. Proc. IEEE, 77 (2), 257-286

[17] Rajashekararadhya, S. V. and Ranjan, P. V., 2009. Support Vector Machine Based Handwritten Numeral Recognition of Kannada Script. IEEE International Advance Computing Conference, pp. 381-386.

[18] Jain, A. K., Duin, R. P. W. and Mao, J., 2000. Statistical Pattern Recognition: A Review. IEEE Transactions on Pattern Analysis and Machine Intelligence, vol. 22, no. 1, pp. 4-37. 